International Journal of Advanced Trends in Computer Science and Engineering

Available Online at http://www.warse.org/IJATCSE/static/pdf/file/ijatcse1581.62019.pdf

https://doi.org/10.30534/ijatcse/2019/1581.62019

\title{
An Improved Framework by Mapping Salient Features for Skin Lesion Detection and Classification using the Optimized Hybrid Features
}

\author{
Rabia Javed ${ }^{1}$, Mohd Shafry Mohd Rahim ${ }^{2}$, Tanzila Saba ${ }^{3}$ \\ ${ }^{1,2}$ School of Computing, Faculty of Engineering, Universiti Teknologi Malaysisa (UTM), Malaysia, 81310 \\ Johor Bharu \\ ${ }^{3}$ College of Computing and Information Science Prince Sultan University, Riyadh, 11586, Saudi Arabia
}

\begin{abstract}
Skin cancer extensively influences the quality of life and it can be dangerous or even lead patients towards death. For the skin lesion analysis, computer-aided systems are very helpful for a dermatologist. To segment, the lesion area is becoming a challenging task even for computer-aided systems due to the various difficulties such as similarities between the lesion and healthy skin area due to the poor contrast, uneven lesion edges, and other artifacts (bubbles, hairs, and ruler marker, etc.). This paper presents an improved saliency-based segmentation method to overcome challenges the current methods. The deep color and PHOG optimized features are passed into the support vector machine (SVM) to classify the images into two categories as benign or melanoma. The classification experiments are accomplished on three datasets termed as ISBI 2016, ISBI 2017, and PH2. The improved accuracies of $90.1 \%, 99.8 \%$, and $98.8 \%$ are attained respectively for each dataset.
\end{abstract}

Key words : Melanoma, Skin lesion, Dermoscopic, Saliency segmentation, Machine learning

\section{INTRODUCTION}

Human skin is not an ordinary thing. It plays a crucial role by shielding the human body from outside elements. According to the current estimation [1], skin cancer is one of the most harmful forms of cancer among the other categories of cancer. Skin cancer depends while the growth of abnormal skin cells cannot be controlled. Skin cancer 'Melanoma' [2] is the much risker type as compared to the other two types as 'Basal Cell Carcinoma', and 'Squamous Cell Carcinoma' [3]. Since Melanoma can easily move from one body organ to another [4]. According to the currently estimated statistics of 2018 [5], an expected 178,560 cases from which 91,270 cases are aggressive in nature.

The dermatologists have inspected the skin with the non-invasive imaging technique called dermatoscopy

[6]. The dermoscopy gave the magnified image through that dermatologist analysis the lesion by utilized the clinical approaches as ABCDE (Asymmetry, Border, Color, Diameter, and Evolving) rules [7], 3-Points checklist, 7Points checklist [8], Menzies, and CASH (Color, Architecture, Symmetry, and Homogeneity) [9]. The problem appears when dermatologist faces the difficulty of the segment the affected area from healthy skin. So, if segmentation is not done properly then it becomes very hard to categorize the lesion into benign and malignant melanoma. Moreover, the current research has presented that the diagnosis of the accuracy rate from the dermatoscopic image is between $75 \%$ to $84 \%$ [10], that is not a very promising percentage. Thus, to overcome this manual problem for skin lesion segmentation and classification the need to come up with automation techniques are very crucial. To segment the lesion many computer-aided systems are present but many of these have low accuracy while some of these fail to work efficiently in varieties of the dermoscopic images as shown in Figure 1. To find differences between the images in Figure 1 are quite hard because all of the images look alike and have minor changes with respect to shape, color, and texture.

\section{Benign}
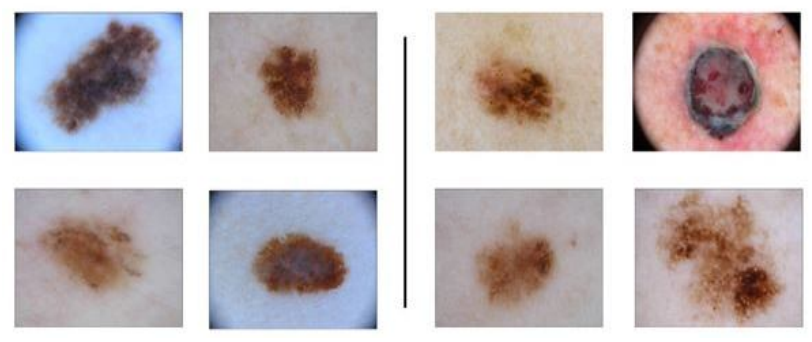

Figure 1: Benign and malignant melanoma

The main contribution of this research is to propose a saliency-based segmentation method combining prominent color channels selected from two color spaces as $\mathrm{LAB}$ [11] and $\mathrm{YCbCr}$ [12], [13]. Moreover, in this paper, the pre-processing phase is dropped to minimize the complexity of the proposed architecture. The proposed segmentation method is able to handle the challenging 
conditions in the images such as artifacts like bubbles, ruler marks, and hairs; Also the low contrast images are tackled by color space transformation. The deep color and PHOG features individually to give good accuracy but after using a hybrid approach of these individual features the combined results of classification are much higher as compared to applied individually.

It has been observed through the literature review, that there are many methods proposed for the analysis of skin lesions, particularly in melanoma diagnosis. In the medical image processing field, the segmentation method/process is considered as the most complex task among the other methods like pre-processing, feature extraction, and classification. For skin lesion segmentation, a huge diversity is present in the approaches such as regionbased approach [14], histogram thresholding [15], and active contour model [16] have shown their importance. In most cases, to improve the irregular lesion border and rough edges the active contour method is used [17].

Currently, the detection of the object from the images by selecting the salient features has been getting great attention from researchers [5], [18], [19]. Saliencybased object identification has split into two categories: the unsupervised model has generated the map directly by getting the details and characteristics of an image. On the other hand the supervised model [5], first extract the numerous features of image regions then it is trained on the labeled dataset after that the saliency map is constructed.

Several previous automated or computer-based methods for lesion extraction and classification have improved their accuracy. Furthermore, in the current study, the researcher has given great devotion to the color space transformation and obtained color correctness. Also, the LAB transformation with saliency-based segmentation [20] for better skin lesion segmentation and classification has improved. A saliency-based method is introduced with the combination of Otsu segmentation for improving segmentation results [21].

In this study, to conduct the experiments three datasets PH2 [22]-[24], ISBI2016 [25], and ISBI2017 [26][28] are selected for demonstrating the validity of our proposed method. First, the proposed improved saliency segmentation method is compared with three existing techniques j-segmentation (JSEG) [29], region-based active contour (RBAC) [30], and previous saliency method. Secondly, the statistics performance measures were determined to highlight the efficiency of the proposed segmentation method. Lastly, after the classification, an experiment has been carried out by comparing the different machine learning classifiers to check the accuracy of the proposed system.

\section{RESEARCH METHOD}

The proposed methodology is based on the three primary phases as shown in Figure 2. The first challenging phase is the extraction of a region of interest (ROI) from dermoscopic images by improving the saliency segmentation method. After that, the extracted deep color, and PHOG features are fused into a feature vector. Then for unique and efficient features selection, the serial-based selection is applied to the fused feature vector. In the final phase, the feature vector is passed to machine learning different classifiers (SVM, KNN, etc). Best performing classifier is selected after conducting the comparative analysis which is based on the performance of statistical measures of all the classifiers [31-32].

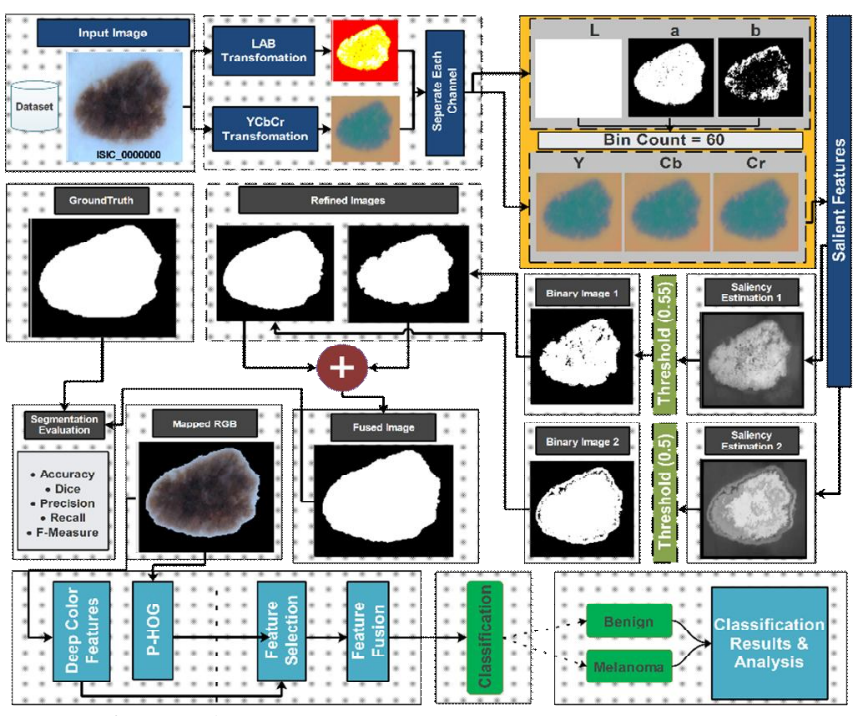

Figure. 2: Proposed methodology flow diagram

\subsection{Lesion Segmentation}

The proposed unsupervised saliency-based segmentation method contains four main parts: color space transformation, salient features, saliency map estimation, and post-processing. In the proposed algorithm's first part is color space transformation the aim of which is to parallel convert the image into a unique color space. This unique color space is abstracted using two color spaces namely $\mathrm{LAB}$, and $\mathrm{YCbCr}$. The second part of the algorithm is to extract the salient features by computing the integral histogram of the image. The third part is the saliency-based mapping estimation by utilizing the salient features. The fourth part is to smooth out and convert the segmented binary image into RGB.

The first part of the color space as mentioned above will be discussed in detail in this paragraph. To obtain the contrast information from the image, two different color spaces including $\mathrm{LAB}$ and $\mathrm{YCbCr}$ are applied. The main advantage of using LAB color space rather than RGB and other color spaces is that LAB color space has a uniform spacing of colors. Also, by the graphical interpretation, the RGB is non-linear while LAB is linear. Moreover, the LAB color space L (luminance factor) calculated from the image have almost same values for all the pixels. Hence, by discarding this values execution time and data size are reduced. 
Rabia Javed et al., International Journal of Advanced Trends in Computer Science and Engineering, 8(1.6), 2019,95 - 101

The salient features are the most prominent features of the affected area present in the image. In the lesion segmentation section, the integral histogram is used for the extraction of the salient features. The obtain matrix has the saliency values with respect to each pixel of the image. The bin count value ' 60 ' is set after the experiments. The minimum and maximum features value of each pixel is calculated for creating the saliency map. After getting the salient feature, two saliency map is estimated. The threshold function is implemented and after performing the experiment on threshold value the finally two value is selected. These grey scale maps are then transformed into a binary image. In this study, the post-processing is constructed by two steps 1) smoothness filter is applied on the binary segmented image where very sharp edges are detected. 2) After that this binary segmented image is mapped into the RGB image.

\subsection{Feature Extraction}

The features extraction/selection/is a primary step as important as the segmentation phase. In feature extraction phase, for this study two types of features: a) deep color, and b) PHOG features are extracted as presented in Figure 3.

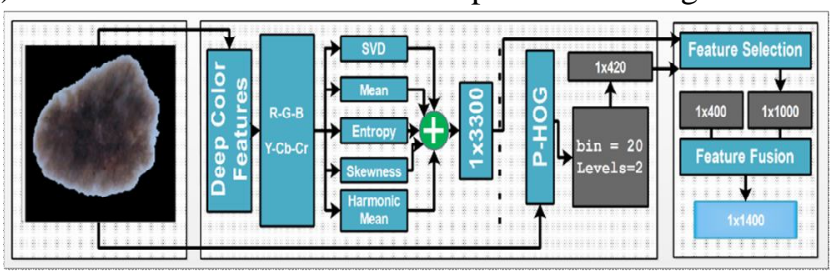

Figure. 3: Feature extraction and finalization

\subsection{Deep Color Features}

For RGB images, first the $\mathrm{YCbCr}$ color space channels are separated then these statistic measures: $\operatorname{SVD}\left(\delta_{i}\right)$, mean $\left(\bar{r}_{)}\right.$, entropy $\left(E_{t_{i}}\right)$, skewness $\left(S k_{i}\right)$, and harmonic( $\left.\quad \lambda_{i}\right)$ means are calculated for each channel. After that $\mathrm{YCbCr}$ transformation is performed for each R, G, and B using the following equations.

$$
\begin{aligned}
& Y=\operatorname{Im}_{R} \cdot R^{\prime}+\operatorname{Im}_{G} \cdot G^{\prime}+\operatorname{Im}_{B} \cdot B^{\prime} \\
& C b=\left[\frac{1}{2} \cdot \frac{B^{\prime}-Y^{\prime}}{1-\operatorname{Im}_{B}}\right] \\
& C r=\left[\frac{1}{2} \cdot \frac{R^{\prime}-Y^{\prime}}{1-\operatorname{Im}_{R}}\right]
\end{aligned}
$$

where $\operatorname{Im}_{R}, \operatorname{Im}_{G}$, and $\operatorname{Im}_{B}$ are ordinarily derived from the definition of the corresponding RGB space, and required to satisfy $I m_{R}+I m_{G}+I m_{B}=1$. So, we have a total of 6 color channels including, R G B, Y, Cb, and $\mathrm{Cr}$. Statistical measures are performed on each channel as summarized in Table 1.
Table 1: Statistical Measure Calculation

\begin{tabular}{cll}
\hline $\begin{array}{c}\text { Sr. } \\
\text { No }\end{array}$ & Statistical Measure & Equation \\
\hline 1 & $\begin{array}{l}\text { Single-Vector } \\
\text { Decomposition } \\
2\end{array}$ & $\delta\left(R_{i * j}\right)=U_{i^{*} i} S_{i^{*} j} V_{j^{*} j}$ \\
3 & $\bar{\gamma}_{i}=\frac{\sum \gamma_{i}}{n_{i}}$ \\
4 & Skean & $E t_{i}=\sum \gamma_{i} * \log \left(\gamma_{i}\right)$ \\
5 & Harmonic Mean & $S k_{i}=\sqrt{\frac{\sum_{j=1}^{N}\left(\gamma_{i}-\bar{\gamma}\right)}{(n-1) y^{3}}}$ \\
& & $\lambda_{i}=\frac{\sum f}{\sum \frac{f}{i}}$ \\
\hline
\end{tabular}

Finally, all statistical measures are fused into a single row for each channel.

$F_{i}=\left[\delta_{i}+\bar{\gamma}_{i}+E t_{i}+S k_{i}+\lambda_{i}\right]$

Where i denotes each channel, here we have 6 color channels.

$$
F v_{1}=\sum_{i=1}^{6} F_{i}
$$

Since we have an imbalanced feature vector for color features, a balanced feature descriptor is constructed using consisting of minimum features for all images using Equation (0.6).

$F v_{1}=N \times\left(\min \left(F v_{1}\right)\right)$

$F v_{1}=N \times 3300$

\subsection{PHOG Features}

After deep color features extraction, shape features are extracted using 20 bins for histogram generation and three- levels of pyramid for features. First, edges are detected using a canny edge detector and hence the pyramid of oriented gradients are calculated using three-level pyramids. As a result of PHOG feature extraction, 420 features are extracted as shown in the following Equation.

$F v_{2}=N \times 420$

\subsection{Feature Selection and Fusion}

Let, $F v_{1}$ and $F v_{2}$ be two random features vectors having redundant values, their mutual information can be formulated as:

$$
I\left(F v_{1}, F v_{2}\right)=\iint p\left(F v_{1}, F v_{2}\right) \cdot \log \frac{p\left(F v_{1}, F v_{2}\right)}{p\left(F v_{1}\right) \cdot p\left(F v_{2}\right)} d x \cdot d y
$$


After the selection of these two feature vectors, the feature decomposition is applied to get the final fused feature vector. Features are sorted in descending order and a technique of feature selection has adopted in such a way that, 1000 features are selected from $F v_{1}$ and 400 are features selected from $F v_{2}$. The final feature vector can be calculated as:

$$
\begin{aligned}
& F V=\sum_{i=1}^{n} F v_{i} \\
& \prod \text { Fused }=\left[\left(F v_{1} \times 1000\right)+\left(F v_{2} \times 400\right)\right]
\end{aligned}
$$

As an output of serial based fusion, a feature vector $(F V \times 1400)$ is obtained.

\subsection{Classification}

$\mathrm{FV}$ is passed to support vector machine classifier and the extracted features are classified using supervised classification. The selected datasets are classified into two classes: benign and malignant melanoma. The experiment details and the results of the classifier are described in the subsequent section.

\section{RESULTS AND ANALYSIS}

All results are evaluated on Core i7 $7^{\text {th }}$ generation laptop has 16 Gigabytes of RAM and a GPU of Radeon R7 having Matlab 18a. To categories the segmented image currently various classification algorithms exist. In this research, the extracted features from the segmented images are evaluated using SVM classifier tested on three different datasets: 1) ISBI 2016, 2) ISBI 2017, and 3) PH2.

\subsection{Segmentation Experiment and Results}

To check the validity of proposed segmentation in this study two types of experiments a) qualitative, and b) quantitative are performed. The detail results of these experiments are further explained.

\subsection{Qualitative Experiment}

However, various segmentation algorithms exist. So, it's quite challenging to compare the proposed segmentation method by all of these segmentation methods. For the qualitative experiment, three online available segmentation methods 1) J-segmentation (JSEG) [32], 2) Region-based active contour (RBAC) [30], and 3) Saliencybased segmentation are compared with our proposed segmentation method as illustrated in Figure.3. Our method accurately identifies the lesion area that is very adjacent to the ground-truth boundary represented as a green color, while the detection of the other four methods is not accurate. As it is clearly seen in the image 'ISBI_0000171' the JSEG fail to detect the lesion area, and the other two methods are unable to extract efficiently. The proposed method extracts the lesion area by achieving $92.15 \%$ accuracy. Some of the images comparisons are represented in Figure 4.

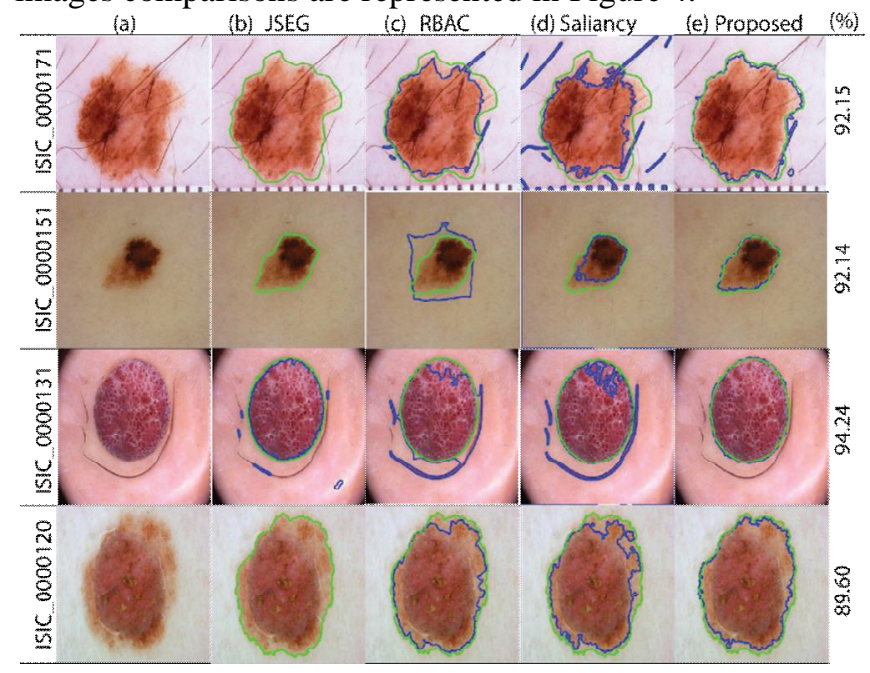

Figure. 4: Proposed segmentation technique comparative analysis with different techniques

\subsection{Quantitative Experiment}

For quantitative evaluation of our proposed method effectiveness, six statistic measures are computed accuracy (Acc.), dice, Jaccard (Jacc), precision (Prec), recall, and Fmeasure. The experiment is performed on more than 2000 images but in this paper, only top ten images are selected and their details results are shown in Table 2.

\begin{tabular}{|c|c|c|c|c|c|c|c|}
\hline \multirow[b]{2}{*}{$\begin{array}{l}\text { Sr. } \\
\text { No. }\end{array}$} & \multirow[b]{2}{*}{ Image No } & \multicolumn{6}{|c|}{$\begin{array}{c}\text { Performance Evaluation Matrices } \\
(\%)\end{array}$} \\
\hline & & $\begin{array}{l}\text { Ac } \\
\text { c. }\end{array}$ & $\begin{array}{l}\text { Di } \\
\text { ce }\end{array}$ & $\begin{array}{l}\mathrm{Jac} \\
\mathrm{c} .\end{array}$ & $\begin{array}{l}\text { Pre } \\
\text { c. }\end{array}$ & $\begin{array}{c}\text { Rec } \\
\text { all }\end{array}$ & $\begin{array}{l}\text { F- } \\
\text { measu } \\
\text { res }\end{array}$ \\
\hline \multirow{2}{*}{1.} & ISBI_0010 & 96. & 0.9 & 0.9 & 0.9 & & \\
\hline & 447 & 9 & 7 & 8 & 7 & 1.00 & 0.98 \\
\hline \multirow{2}{*}{2.} & ISBI_0000 & 96. & 0.9 & 0.9 & 0.9 & & \\
\hline & 185 & 8 & 7 & 8 & 8 & 0.99 & 0.98 \\
\hline \multirow{2}{*}{3.} & ISBI_0000 & 96. & 0.9 & 0.9 & 1.0 & & \\
\hline & 029 & 6 & 7 & 8 & 0 & 0.97 & 0.98 \\
\hline \multirow{2}{*}{4.} & ISBI_0000 & 96. & 0.9 & 0.9 & 1.0 & & \\
\hline & 543 & 3 & 6 & 8 & 0 & 0.97 & 0.98 \\
\hline \multirow{2}{*}{5.} & ISBI_0000 & 96. & 0.9 & 0.9 & 1.0 & & \\
\hline & 065 & 2 & 6 & 8 & 0 & 0.96 & 0.98 \\
\hline \multirow{2}{*}{6.} & ISBI_0000 & 95. & 0.9 & 0.9 & 0.9 & & \\
\hline & 104 & 3 & 5 & 8 & 7 & 0.98 & 0.98 \\
\hline \multirow{2}{*}{7.} & ISBI_0000 & 95. & 0.9 & 0.9 & 1.0 & & \\
\hline & 093 & 2 & 5 & 8 & 0 & 0.95 & 0.98 \\
\hline \multirow{2}{*}{8.} & ISBI_0010 & 95. & 0.9 & 0.9 & 0.9 & & \\
\hline & 251 & 0 & 5 & 7 & 9 & 0.96 & 0.97 \\
\hline \multirow{2}{*}{9.} & ISBI_0000 & 94. & 0.9 & 0.9 & 0.9 & & \\
\hline & 097 & 7 & 5 & 7 & 9 & 0.96 & 0.97 \\
\hline \multirow{4}{*}{10.} & ISBI_0000 & 94. & 0.9 & 0.9 & 0.9 & & \\
\hline & 001 & 4 & 4 & 7 & 6 & 0.99 & 0.97 \\
\hline & Average & 95. & 0.9 & 0.9 & 0.9 & & \\
\hline & & 7 & 5 & 7 & 8 & 0.97 & 0.97 \\
\hline
\end{tabular}

Table 2: Proposed Segmentation Method Detail Results 


\subsection{Classification Experiment and Results}

The performance assessment was accomplished using 10-fold cross-validation. The five evaluation matrices: precision(Prec.), sensitivity(Sens.), specificity(Spec.), the area under the curve(AUC), and accuracy (Acc.) are used for the validity of classification results. The comparison is performed between the achieved accuracies by these three datasets on the SVM classifier. As it can be seen in Table 3, the highest accuracy achieved is $99.8 \%$ on ISBI 2017 dataset. The proposed fused features vector also performed well on the $\mathrm{PH} 2$ dataset by gaining $98.9 \%$ accuracy. The

Table 3: Classification Detail Results

\begin{tabular}{|c|c|c|c|c|c|c|c|c|}
\hline \multirow{2}{*}{$\begin{array}{c}\text { Datase } \\
\text { ts }\end{array}$} & \multicolumn{3}{|c|}{ Extracted Features } & \multicolumn{5}{|c|}{$\begin{array}{c}\text { Performance Evaluation } \\
\text { Matrices }(\%)\end{array}$} \\
\hline & $\begin{array}{l}\text { Col } \\
\text { or }\end{array}$ & $\begin{array}{c}\mathrm{PHO} \\
\mathrm{G}\end{array}$ & $\begin{array}{c}\text { Fus } \\
\text { ed }\end{array}$ & $\begin{array}{c}\text { Pre } \\
\text { c. }\end{array}$ & $\begin{array}{l}\text { Se } \\
\text { ns. }\end{array}$ & $\begin{array}{c}\text { Spe } \\
\text { c. }\end{array}$ & $\begin{array}{c}\mathrm{Au} \\
\mathrm{C}\end{array}$ & $\begin{array}{r}\text { Ac } \\
\text { c. }\end{array}$ \\
\hline \multirow{5}{*}{ 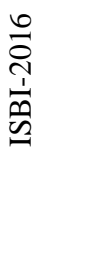 } & $\checkmark$ & & & 82 & 82. & 0.8 & 0.8 & 82. \\
\hline & & & & & 1 & 1 & 1 & 1 \\
\hline & & $\checkmark$ & & 89 & $\begin{array}{c}89 . \\
5\end{array}$ & $\begin{array}{c}0.8 \\
9\end{array}$ & $\begin{array}{c}0.8 \\
9\end{array}$ & $\begin{array}{c}89 . \\
9\end{array}$ \\
\hline & & & $\checkmark$ & 90 & $\begin{array}{c}90 . \\
1\end{array}$ & $\begin{array}{c}0.9 \\
0\end{array}$ & $\begin{array}{c}0.9 \\
4\end{array}$ & $\begin{array}{c}90 . \\
1\end{array}$ \\
\hline & $\checkmark$ & & & 91 & 91. & 0.9 & 0.9 & 91. \\
\hline \multirow{5}{*}{ 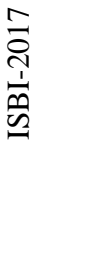 } & & & & & 5 & 1 & 4 & 1 \\
\hline & & $\checkmark$ & & 99 & 99 & 1.0 & 1.0 & 99. \\
\hline & & & & & & & 0 & 7 \\
\hline & & & $\checkmark$ & 99 & 99 & 1.0 & $\begin{array}{c}1.0 \\
0\end{array}$ & $\begin{array}{c}99 . \\
8\end{array}$ \\
\hline & $\checkmark$ & & & $\begin{array}{c}95 . \\
6\end{array}$ & $\begin{array}{c}95 . \\
3\end{array}$ & $\begin{array}{c}0.9 \\
5\end{array}$ & $\begin{array}{c}0.9 \\
8\end{array}$ & $\begin{array}{c}95 . \\
5\end{array}$ \\
\hline \multirow[t]{2}{*}{$\stackrel{I}{I}$} & & $\checkmark$ & & 96 & $\begin{array}{c}96 . \\
6\end{array}$ & $\begin{array}{c}0.9 \\
6\end{array}$ & $\begin{array}{c}0.9 \\
9\end{array}$ & $\begin{array}{c}96 . \\
6\end{array}$ \\
\hline & & & $\checkmark$ & $\begin{array}{c}98 . \\
6\end{array}$ & $\begin{array}{c}98 . \\
6\end{array}$ & $\begin{array}{c}0.9 \\
8\end{array}$ & $\begin{array}{c}1.0 \\
0\end{array}$ & $\begin{array}{c}98 . \\
8\end{array}$ \\
\hline
\end{tabular}

ISBI 2016 dataset also gained good accuracy of $90.1 \%$.

The SVM results have been achieved on three datasets ISBI 2016, ISBI 2017, and PH2 presented in the confusion matrixes as shown in Figure. 5. These confusion matrixes give an in-depth inside about the trained classifier. As it can be observed that $\mathrm{PH} 2$ have three classes: atypical nevus, common nevus, and melanoma as the other two datasets have only two classes: benign and malignant. The selected features also efficiently classify the $\mathrm{PH} 2$ dataset into three classes.

\section{CONCLUSION}

An accurate skin lesion area extraction is imperative to efficiently categorize the benign and malignant melanoma. The performance of the classification phase is highly depended on the accurately segmented images. In this article, an improved version of the saliencybased method is proposed by adding the two different color spaces. Through combining the $\mathrm{LAB}$ and $\mathrm{YCbCr}$, the results of the segmentation process are enhanced. A comparison is done between the proposed segmentation method and the earlier methods to demonstrate the contribution and validity of this research. The results obtained from the proposed segmentation method are very close to the ground truth images with maximum accuracy. In the future, lesion segmentation can be further improved by extracting more accurate minimum features and by reducing the system time.

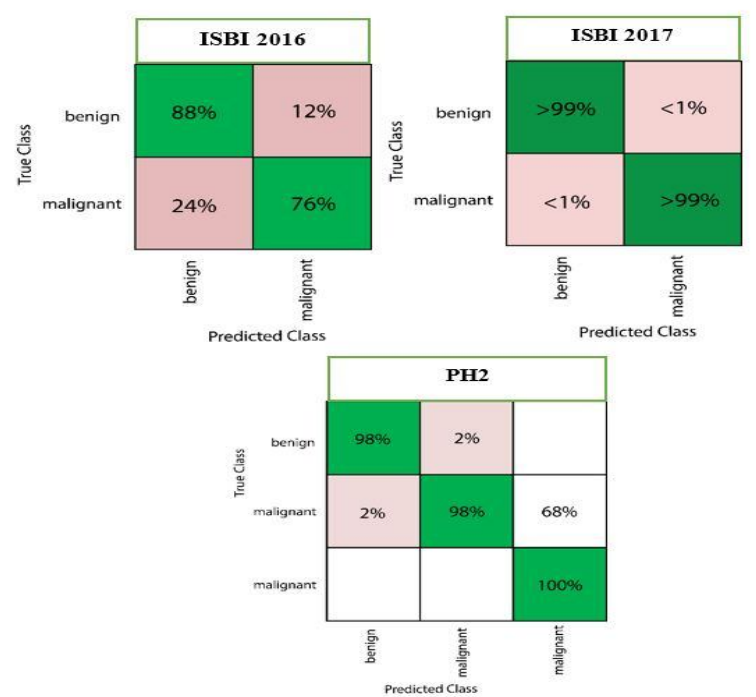

Figure. 5: Confusion matrixes of three datasets ISBI 2016, ISBI 2017, and PH2

\section{ACKNOWLEDGEMENTS}

We appreciatively recognize the technical support and research facilities provided by the Universiti Teknologi Malaysia (UTM), without which this research has not been accomplished. This work was also supported by Artificial Intelligence and Data Analytics (AIDA) Lab Prince Sultan University Riyadh Saudi Arabia. Authors are thankful for the support.

\section{REFERENCES}

1. "Leading Sites of New Cancer Cases and Deaths 2010 Estimates," Health Policy (New. York)., vol. 730, pp. 2010-2010, 2010.

2. A. cancer Society, "What Is Melanoma Skin Cancer? Types of skin cells," pp. 1-5, 2018.

3. American Cancer Society, "Skin Cancer: Basal and Squamous Cell What are basal and squamous cell skin cancers ?," Am. Cancer Soc., pp. 1-10, 2011.

4. M. Janda and H. P. Soyer, "Using Advances in Skin Imaging Technology and Genomics for the Early Detection and Prevention of Melanoma," Dermatology, vol. 235, no. 1, pp. 1-3, 2018.

https://doi.org/10.1159/000493260

5. R. This, C. C. Attribution-noncommercial-noderivs, C. C. By-nc-nd, T. If, and W. Rose, "Supervised Saliency Map Driven Segmentation of Lesions in Dermoscopic Images," no. August 2018, 2015.

6. I. Zalaudek et al., "Dermoscopy in general dermatology," Dermatology, vol. 212, no. 1, pp. 7-18, 
Rabia Javed et al., International Journal of Advanced Trends in Computer Science and Engineering, 8(1.6), 2019,95 - 101

2005. https://doi.org/10.1159/000089015

7. A. Gola Isasi, B. García Zapirain, and A. Méndez Zorrilla, "Melanomas non-invasive diagnosis application based on the $\mathrm{ABCD}$ rule and pattern recognition image processing algorithms," Comput. Biol. Med., vol. 41, no. 9, pp. 742-755, 2011.

8. T. Y. Satheesha, D. Satyanarayana, M. N. G. Prasad, and K. D. Dhruve, "Melanoma Is Skin Deep: A 3D Reconstruction Technique for Computerized Dermoscopic Skin Lesion Classification," IEEE J. Transl. Eng. Heal. Med., vol. 5, no. December 2016, 2017. https://doi.org/10.1109/JTEHM.2017.2648797

9. T. Majtner, S. Yildirim-Yayilgan, and J. Y. Hardeberg, "Combining deep learning and hand-crafted features for skin lesion classification," 2016 6th Int. Conf. Image Process. Theory, Tools Appl. IPTA 2016, 2017. https://doi.org/10.1109/IPTA.2016.7821017

10. J. A. A. Salido and C. R. Jr., "Using Deep Learning for Melanoma Detection in Dermoscopy Images," Int. J. Mach. Learn. Comput., vol. 8, no. 1, pp. 61-68, 2018.

11. R. Garnavi, M. Aldeen, M. E. Celebi, A. Bhuiyan, C. Dolianitis, and G. Varigos, "Skin Lesion Segmentation Using Color Channel Optimization and Clusteringbased Histogram," Mach. Learn., vol. 3, no. 12, 2009.

12. H. Nisar, Y. K. Ch'ng, T. Y. Chew, V. V. Yap, K. H. Yeap, and J. J. Tang, "A color space study for skin lesion segmentation," ICCAS 2013 - 2013 IEEE Int. Conf. Circuits Syst. "Advanced Circuits Syst. Sustain., pp. 172-176, 2013.

13. M. Saber Iraji and A. Tosinia, "Skin Color Segmentation in YCBCR Color Space with Adaptive Fuzzy Neural Network (Anfis)," Int. J. Image, Graph. Signal Process., vol. 4, no. 4, pp. 35-41, 2012.

14. H. Iyatomi et al., "Fast and accurate border detection in dermoscopy images using statistical region merging," Med. Imaging 2007 Image Process., vol. 6512, no. 3, p. 65123V, 2007.

15. M. Niewczas, W. Maly, and A. Strojwas, "Short Papers," vol. 18, no. 4, pp. 494-501, 1999. https://doi.org/10.1109/43.752932

16. R. Kasmi, K. Mokrani, R. K. Rader, J. G. Cole, and W. V. Stoecker, "Biologically inspired skin lesion segmentation using a geodesic active contour technique," Ski. Res. Technol., vol. 22, no. 2, pp. 208222, 2016. https://doi.org/10.1111/srt.12252

17. A. F. De Araujo, A. S. Pereira, N. Marranghello, B. Rossetti, and J. M. R. S. Tavares, "Hybrid methodology to segment skin lesions based on active contour and region growing techniques," no. May 2014.

18. K. Hu et al., "Automatic segmentation of dermoscopy images using saliency combined with adaptive thresholding based on wavelet transform," Multimed. Tools Appl., 2019.

19. A. Borji, M. M. Cheng, H. Jiang, and J. Li, "Salient
Object Detection: A Benchmark," IEEE Trans. Image

Process., vol. 24, no. 12, pp. 5706-5722, 2015.

https://doi.org/10.1109/TIP.2015.2487833

20. M. A. Khan et al., "Construction of saliency map and hybrid set of features for efficient segmentation and classification of skin lesion," Microsc. Res. Tech., no. August 2018, pp. 1-23, 2019.

21. Z. Jiang, Y. Li, H. Fan, J. Liu, and F. Xie, "Automatic segmentation of dermoscopy images using saliency combined with Otsu threshold," Comput. Biol. Med., vol. 85, no. March, pp. 75-85, 2017.

22. M. Ruela, C. Barata, J. S. Marques, and J. Rozeira, "A system for the detection of melanomas in dermoscopy images using shape and symmetry features," Comput. Methods Biomech. Biomed. Eng. Imaging Vis., vol. 5, no. 2, pp. 127-137, 2017. https://doi.org/10.1080/21681163.2015.1029080

23. M. Nasir, M. Attique Khan, M. Sharif, I. U. Lali, T. Saba, and T. Iqbal, "An improved strategy for skin lesion detection and classification using uniform segmentation and feature selection based approach," Microsc. Res. Tech., no. December 2017, 2018.

24. Y. Yuan, M. Chao, and Y. Lo, "Deep Fully Convolutional Networks With Jaccard Distance," vol. 36, no. 9, pp. 1876-1886, 2017. https://doi.org/10.1109/TMI.2017.2695227

25. M. Aljanabi, Y. E. Özok, J. Rahebi, and A. S. Abdullah, "Skin lesion segmentation method for dermoscopy images using artificial bee colony algorithm," Symmetry (Basel)., vol. 10, no. 8, 2018.

26. X. Li, J. Wu, H. Jiang, and E. Z. Chen, "Skin Lesion Classification Via Combining Deep Learning Features and Clinical Criteria Representations," 2018.

27. S. Chatterjee, D. Dey, and S. Munshi, "Optimal selection of features using wavelet fractal descriptors and automatic correlation bias reduction for classifying skin lesions," Biomed. Signal Process. Control, vol. 40, pp. 252-262, 2018. https://doi.org/10.1016/j.bspc.2017.09.028

28. M. A. Al-masni, M. A. Al-antari, M. T. Choi, S. M. Han, and T. S. Kim, "Skin lesion segmentation in dermoscopy images via deep full resolution convolutional networks," Comput. Methods Programs Biomed., vol. 162, pp. 221-231, 2018. https://doi.org/10.1016/j.cmpb.2018.05.027

29. M. E. Celebi et al., "A methodological approach to the classification of dermoscopy images," vol. 6, no. 6, pp. 247-253, 2009.

30. T. F. Chan and L. A. Vese, "Donald Middleton, L.D.S.," Br. Dent. J., vol. 10, no. 2, pp. 266-277, 1977.

31. Dhariwal, Sumit, and Sellappan Palaniappan. "An Efficient Approach for Semantic Image Classification Using Normalization Method." International Journal of Advanced Trends in Computer Science and Engineering, vol. 8, no. 4, 2019, pp. 1268-74. https://doi.org/10.30534/ijatcse/2019/37842019 
Rabia Javed et al., International Journal of Advanced Trends in Computer Science and Engineering, 8(1.6), 2019, 95 - 101

32. Nasharuddin, Nurul Amelina, et al. "Multi-Feature Vegetable Recognition Using Machine Learning Approach on Leaf Images." International Journal of Advanced Trends in Computer Science and Engineering, vol. 8, no. 4, 2019, pp. 1789-94. https://doi.org/10.30534/ijatcse/2019/110842019

33. Y. Deng and B. S. Manjunath, "Unsupervised segmentation of color-texture regions in images and video," IEEE Trans. Pattern Anal. Mach. Intell., vol. 23, no. 8, pp. 800-810, 2001.

https://doi.org/10.1109/34.946985 